\title{
Synchronous Occurrence of Mycosis Fungoides, Diffuse Large B Cell Lymphoma and Acute Myeloid Leukemia
}

\author{
Junichi Miyatake ${ }^{1}$, Hiroaki Inoue ${ }^{2}$, Kentarou Serizawa ${ }^{2}$, Yasuyoshi Morita ${ }^{2}$, JL Espinoza ${ }^{2}$, \\ Hirokazu Tanaka ${ }^{2}$, Takahiro Shimada ${ }^{2}$, Yoichi Tatsumi ${ }^{2}$, \\ Takashi Ashida $^{2}$ and Itaru Matsumura ${ }^{2}$
}

\begin{abstract}
:
Patients with mycosis fungoides (MF), the most common subtype of primary cutaneous T-cell lymphoma, have an increased risk of developing secondary malignancies. We herein report two rare cases of MF concurring with diffuse large B cell lymphoma (B lymphoid lineage) and acute myeloid leukemia (myeloid lineage) in two otherwise healthy elderly patients. Potential etiologic factors, including the impact of the therapyassociated inflammatory response on the development of secondary tumors in patients with MF, are discussed. Further clinical, experimental and genetic studies are needed to elucidate possible physiopathogenic associations among the three concurrent malignancies occurring in the cases presented here.
\end{abstract}

Key words: mycosis fungoides, diffuse large B-cell lymphoma, acute myelomonocytic leukemia, TET2

(Intern Med 57: 1445-1453, 2018)

(DOI: 10.2169/internalmedicine.9668-17)

\section{Introduction}

Mycosis fungoides (MF) is the most common subtype of primary cutaneous T-cell lymphoma (CTCL), resulting from the clonal proliferation of skin infiltrating T helper-like lymphocytes (1). Clinically, MF is characterized by scaly, erythematous skin plaques, frequently having a long natural history, with a median duration from the onset to the diagnosis of six years (2). Approximately $30 \%$ of patients with MF have generalized erythroderma or skin tumors $(2,3)$. Notably, patients with CTCL are at high risk for developing secondary malignancies, including hematological malignancies $(4,5)$.

Diffuse large B-cell lymphoma (DLBCL) is the most common type of Non-Hodgkin lymphoma in adults. DLBCL typically affects older individuals, with a median age of approximately 70 at the diagnosis (6). DLBCL frequently arises from the transformation of normal B cells in lymph nodes or spleen, but transformation from other hematological malignancies has also been reported (7).
Acute myeloid leukemia (AML) is the most common subtype of acute leukemia in adults. This highly heterogeneous disease originates from the abnormal and uncontrolled proliferation of hematopoietic precursor cells that results in the accumulation of immature myeloid cells in the bone marrow (8). AML more frequently affects older individuals and is associated with disease relapse and a poor prognosis. AML (M4) is a subtype of AML, according to the French America British (FAB) classification, that involves the proliferation of myeloblasts and is classified as 'AML not otherwise classified' under the WHO system. This entity represents $5-10 \%$ of AML cases in adults and nearly $3 \%$ of all pediatric leukemias (9).

We herein report two extremely rare cases of three hematological malignancies occurring on both lymphoid lineages and myeloid lineage.

\section{Targeted capture sequencing of Ten-eleven translo-} cation 2 (TET2)

Targeted sequence screening for the TET2 gene mutation was performed in three tissues of Case 1: skin (the lesion of

${ }^{1}$ Department of Hematology, Sakai Hospital Kindai University Faculty of Medicine, Japan and ${ }^{2}$ Department of Hematology and Rheumatology, Kindai University Faculty of Medicine, Japan

Received: June 19, 2017; Accepted: October 15, 2017; Advance Publication by J-STAGE: January 11, 2018

Correspondence to Dr. Junichi Miyatake, j-miyatake@shiroyama-hsp.or.jp 


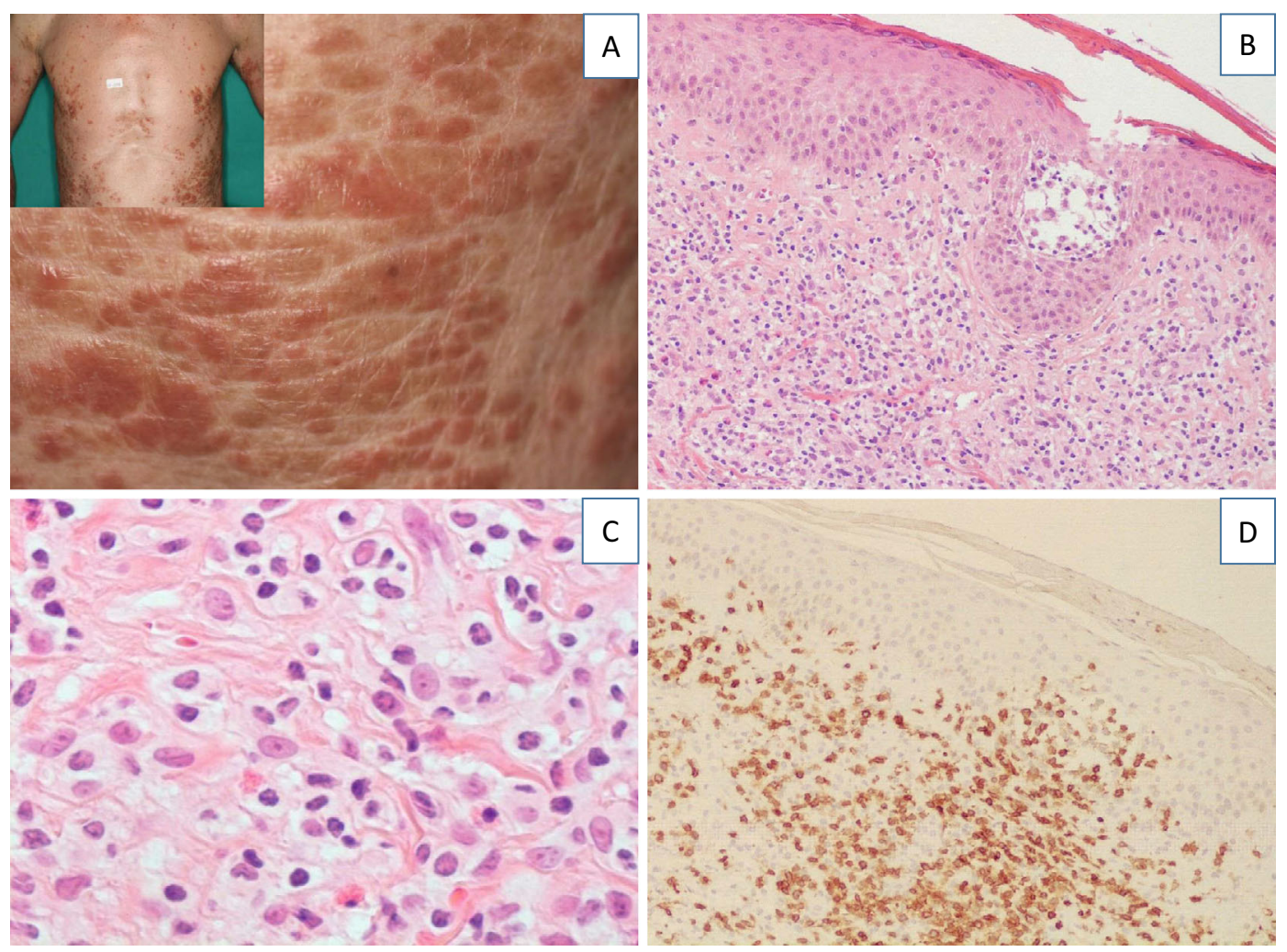

Figure 1. Case 1, mycosis fungoides. (A) Pruritic erythematous skin lesions with moderate hyperpigmentation over the abdomen that extended to the thorax. (B) A biopsy specimen from the skin showing the infiltration of epidermotropic lymphoid cells with a collection of malignant lymphocytes in the epidermis forming a Pautrier's micro abscess. Epidermotropism is also visible. Hematoxylin and Eosin $(H \& E)$ staining $(\times 100)$. (C) Small and medium atypical lymphocytes in the superficial dermis $(\mathrm{H} \& \mathrm{E}$ staining $\times 1,000)$. (D) $\mathrm{CD3}^{+}$cells are visible throughout the extensive lymphocytic infiltrate in the superficial dermis (CD3 stain, $\times 100)$.

$\mathrm{MF}$ ), spleen (the lesion of DLBCL), and oral mucosa (germ line control).

Genomic DNA (gDNA) from paraffin-embedded skin tissue was extracted with a QiAamp DNA FFPE Tissue Kit (Qiagen, Hilden, Germany), and gDNA from cryopreserved spleen tissue and freshly obtained oral mucosa were extracted using a Wizard genomic DNA purification Kit (Promega, Madison, USA). After determining the quality and quantity of the extracted gDNA with the PicoGreen dsDNA assay kit (Life Technologies, Palo Alto, USA), the samples were stored at $-20^{\circ} \mathrm{C}$ until analysis.

A next-generation sequencing (NGS) assay for the TET2 gene mutation was designed to detect mutations in codons 3-14 using an Ion Torrent PGM instrument (Life Technologies). The polymerase chain reaction (PCR) primers for this assay were designed using the Ion Ampliseq designer v.3.4 (Thermo Fisher Scientific, Waltham, USA), and library preparation was performed with $20 \mathrm{ng}$ of genomic DNA using the Ion Ampliseq Library Kit (Life Technologies) according to the manufacturer's instructions. The Ion Xpress Barcode Adapters (Life Technologies) were ligated into the PCR products and purified with Agencourt Ampure XP beads (Beckman Coulter, Fullerton, USA). Purified libraries were sequenced on an Ion Torrent PGM device (Life Tech- nologies) using the Ion PGM 200 sequencing Kit v2 and the Ion318 v2 Chip Kit. DNA sequencing data were accessed through the Torrent Suite v4.2 software program (Thermo Fisher Scientific). The reads were aligned against the hg19 human reference genome, and the variants were called using the plug-in Variant Caller (ver. 4.2; Life Technologies).

\section{Case Reports}

\section{Case 1}

A 77-year-old man was referred to our hospital with pruritic erythematous skin lesions over most of his body present for more than 2 years (Fig. 1A). He denied symptoms of a fever, fatigue, dyspnea or night sweats. A physical examination revealed rash-like patches, itchy skin and small skin tumors with patches and papules covering $\geq 10 \%$ of the skin surface. A skin biopsy revealed the presence of epidermotropic lymphoid cells that were in association with Pautrier's microabscesses (Fig. 1B). These lymphoid cells stained positive for CD3 and CD4 and were negative for CD20 (Fig. 1C and D) as well as for CD7 and CD8 (data not shown). In addition, these cells were positive for $\mathrm{T}$ cell receptor (TCR) gene rearrangement $(\mathrm{V} \beta / \mathrm{J} \beta 2, \mathrm{D} \beta / \mathrm{J} \beta 1,2)$, as 


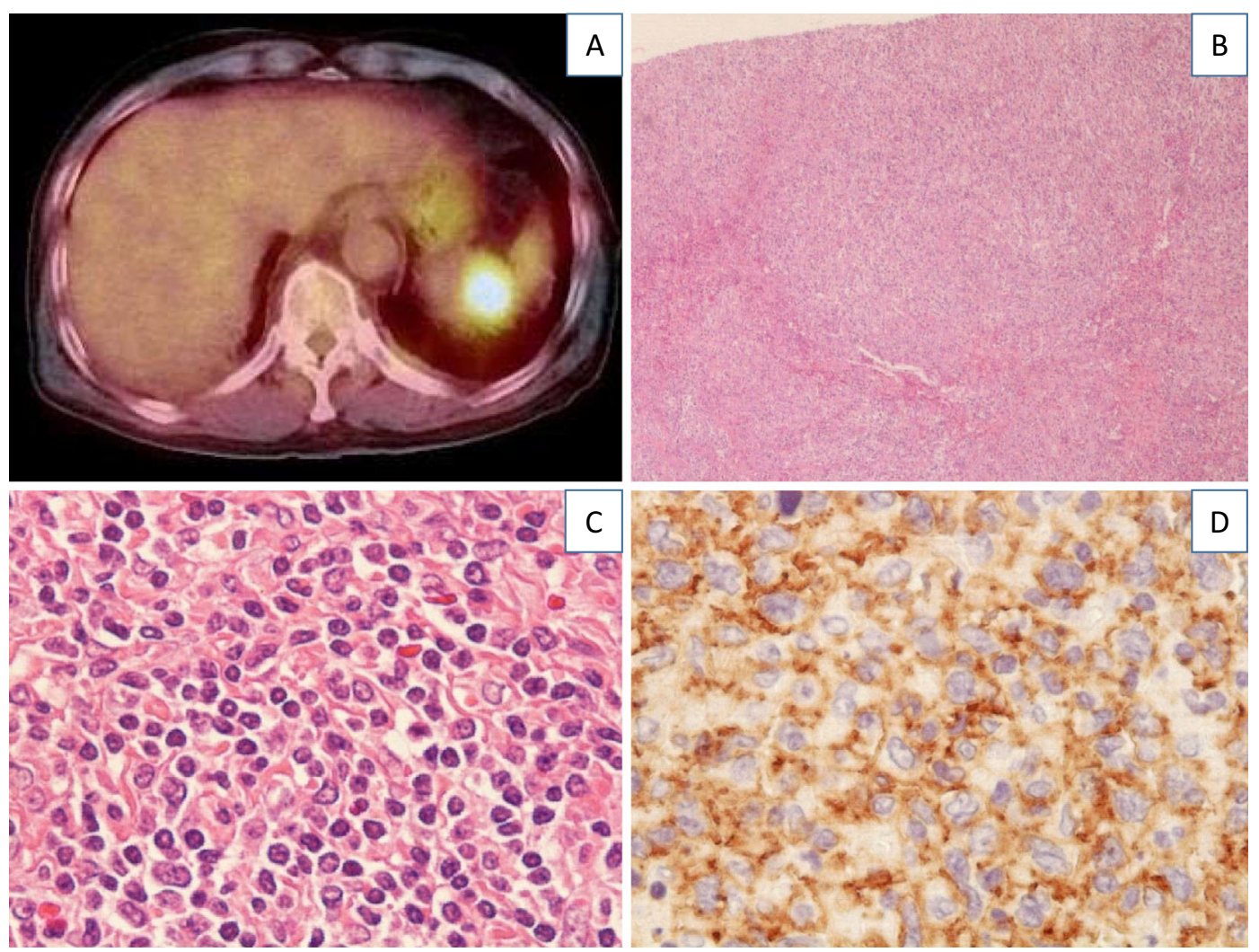

Figure 2. Case 1, DLBCL in the spleen. (A) A space-occupying lesion (SOL) with the strong accumulation of FDG in the spleen (SUV max. 7.23) as observed on PET-CT. Hematoxylin and Eosin (H\&E) staining, of the splenic specimen showing abundant diffuse proliferation of malignant lymphocytes $(B$ : $H \& E$ staining $\times 40, C$ : $H \& E$ staining $\times 400)$. (D) An immunohistochemistry analysis of the spleen specimen showing extensive infiltration of large lymphocytic cells that were positive for CD20, consistent with the diagnosis of DLBCL $(\times 400)$.

confirmed by PCR (data not shown). A diagnosis of MF (infiltrative stage, T2NOMOB0; stage 1B) was therefore made.

The patient underwent narrow-band ultraviolet $\mathrm{B}$ (NBUVB) therapy in combination with a topical steroid, which induced only a partial clinical response. Enhanced computed tomography (CT) of the abdomen performed 12 months after the diagnosis revealed the presence of a space-occupying lesion (SOL) in the spleen. Positron emission tomographyCT (PET-CT) performed at the same time demonstrated an increase uptake of fludeoxyglucose (FDG) involving the splenic SOL (SUVmax. 7.23, Fig. 2A). A pathological examination of a splenic specimen obtained by splenectomy showed the presence of diffuse proliferating large cells that were strongly positive for CD20 (Fig. 2B-D). The patient was therefore diagnosed with DLBCL coexisting with MF.

He was treated with rituximab at $375 \mathrm{mg} / \mathrm{m}^{2}$ on day 1 in combination with the $\mathrm{CHOP}$ regimen (cyclophosphamide $750 \mathrm{mg} / \mathrm{m}^{2}$ on day 1 , doxorubicin $50 \mathrm{mg} / \mathrm{m}^{2}$ on day 1 , vincristine $1.4 \mathrm{mg} / \mathrm{m}^{2}$ on day 1 , and prednisone $60 \mathrm{mg} / \mathrm{m}^{2}$ on days 1-5 and repeated every 21 days), which was given for 6 courses and resulted in the induction of a complete response (CR). Notably, the cutaneous manifestations of MF were significantly improved after 6 courses of R-CHOP treatment, suggesting that the large doses of prednisone (to- tal dose of $360 \mathrm{mg} / \mathrm{m}^{2}$ ), which were used as part of the treatment for DLBCL, may have contributed to the improvement in the skin lesions of MF (Fig. 3A). The patient showed no sign of disease during the subsequent follow-up period, but a routine blood analysis performed at six months after achieving CR revealed severe leukocytosis [white blood cell (WBC) count $115 \times 10^{3} / \mu \mathrm{L}$ with $34 \%$ of monocytoid cells], thrombocytopenia (platelet count $6.8 \times 10^{4} / \mu \mathrm{L}$ ), with no evidence of anemia (red blood cells count $347 \times 10^{4} / \mu \mathrm{L}$ ). An analysis of a bone marrow aspirate showed hypercellular marrow with $4.4 \%$ myeloblasts and $56.6 \%$ monoblasts, which were positive for peroxidase (Fig. 3B and C), naphthol AS-D chloroacetate esterase and alpha-naphthyl butyrate stains (Fig. 3D). These leukemic cells were positive for CD11b, CD13, CD14, CD15, CD33, CD36 and CD64 on flow cytometry. The patient was thus diagnosed with acute myelomonocytic leukemia (FAB classification: AML M4) as the third concurrent hematologic malignancy.

A karyotypic analysis of the leukemic cells revealed a normal karyotype. He was treated with induction chemotherapy for remission that included cytarabine continuously 100 $\mathrm{mg} / \mathrm{m}^{2}$ on days $1-7$ and daunorubicin $50 \mathrm{mg} / \mathrm{m}^{2}$ on days $1-5$, resulting in $\mathrm{CR}$. The patient is currently under $\mathrm{CR}$ for all three hematological malignancies. 


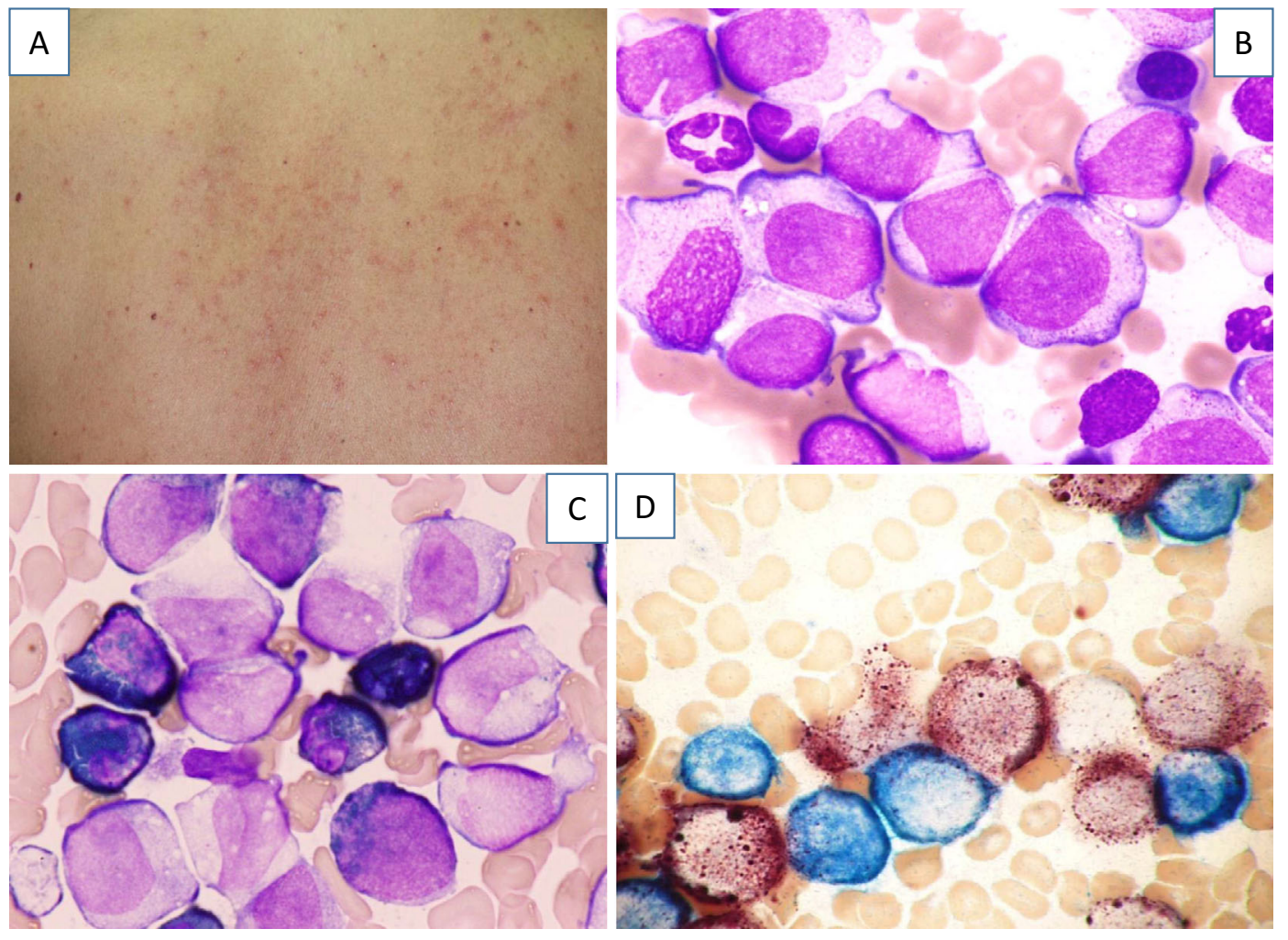

Figure 3. Case 1, cutaneous manifestation of MF and acute myelomonocytic leukemia: (A) The cutaneous manifestations of MF were significantly improved after the six courses of R-CHOP treatment. (B) Bone marrow infiltration by monocytic blasts and myeloid blasts (May-Grunwald-Gimsa staining $\times 1,000)$. (C) The blasts were positive for myeloperoxidase (MPO). (D) Double staining for esterase revealing naphthol AS-D chloroacetate-positive myeloblasts and alpha-naphthyl butyratepositive monoblasts.

Given the asynchronous occurrence of hematological malignancies arising from three different lineages in the same individual, we considered the possibility that these malignancies might have a common origin. We examined potential mutational changes in the TET2 gene in the malignantderived tissues using targeting NGS, since the TET2 gene is frequently mutated in malignancies from the $\mathrm{B}, \mathrm{T}$ and myeloid lineages. Because AML cells were not available for this study, we performed the NGS assay with gDNA samples extracted from the skin (MF) and spleen (DLBCL). Surprisingly, no somatic mutations in the TET2 gene were identified in the malignant tissue analyzed.

\section{Case 2}

A 77-year-old woman was evaluated at another hospital for a $10-\mathrm{cm}$ skin erythema lesion with squama on the left lower abdomen, without itching or pain (Fig. 4A). The patient had no relevant history, except for DLBCL diagnosed 10 years before the admission that had been completely eradicated after splenectomy together with R-CHOP therapy. Biopsy studies and immunohistochemistry of the skin specimens revealed intense cellular infiltrate composed of $\mathrm{CD}^{+}$ and $\mathrm{CD}^{+}, \mathrm{CD} 20$ and $\mathrm{CD} 79 \mathrm{a}^{-}$atypical lymphoid cells with an irregular nucleus (Fig. 4B and C). The patient was therefore diagnosed with MF (Patch stage, T1N0M0B0; stage
1A).

She was treated with topical corticosteroid as monotherapy for four months. Two months later, swelling lymph nodes located in her left cubital fossa were noticed (Fig. 5A), and an analysis by PET-CT indicated the strong accumulation of FDG (SUVmax. 6.92, Fig. 5B). Hematoxylin and Eosin (H \& E) staining of specimens derived from the cubital lymph node showed diffuse proliferating large lymphoid cells (Fig. 5C) that were positive for CD20 (Fig. 5D). She was diagnosed with DLBCL and was therefore treated with the R-CHOP regimen. However, 2 months after starting this therapy, peripheral blood tests revealed WBC counts of $75.2 \times 10^{3} / \mu \mathrm{L}$, with $72 \%$ blasts, hemoglobin $6.6 \mathrm{~g} / \mathrm{dL}$ and platelets $2.1 \times 10^{4} / \mu \mathrm{L}$. An analysis of a bone marrow aspirate showed hypercellular marrow with $77.4 \%$ myeloblasts and monoblasts that were weakly positive for both peroxidase and naphthol AS-D chloroacetate esterase (data not shown). She was diagnosed with concurrent AML (FAB classification: M4). The AML cells possessed a normal karyotype, as assessed by a chromosomal analysis. The patient was transferred to another hospital to undergo special treatment for AML but eventually died from sepsis. 

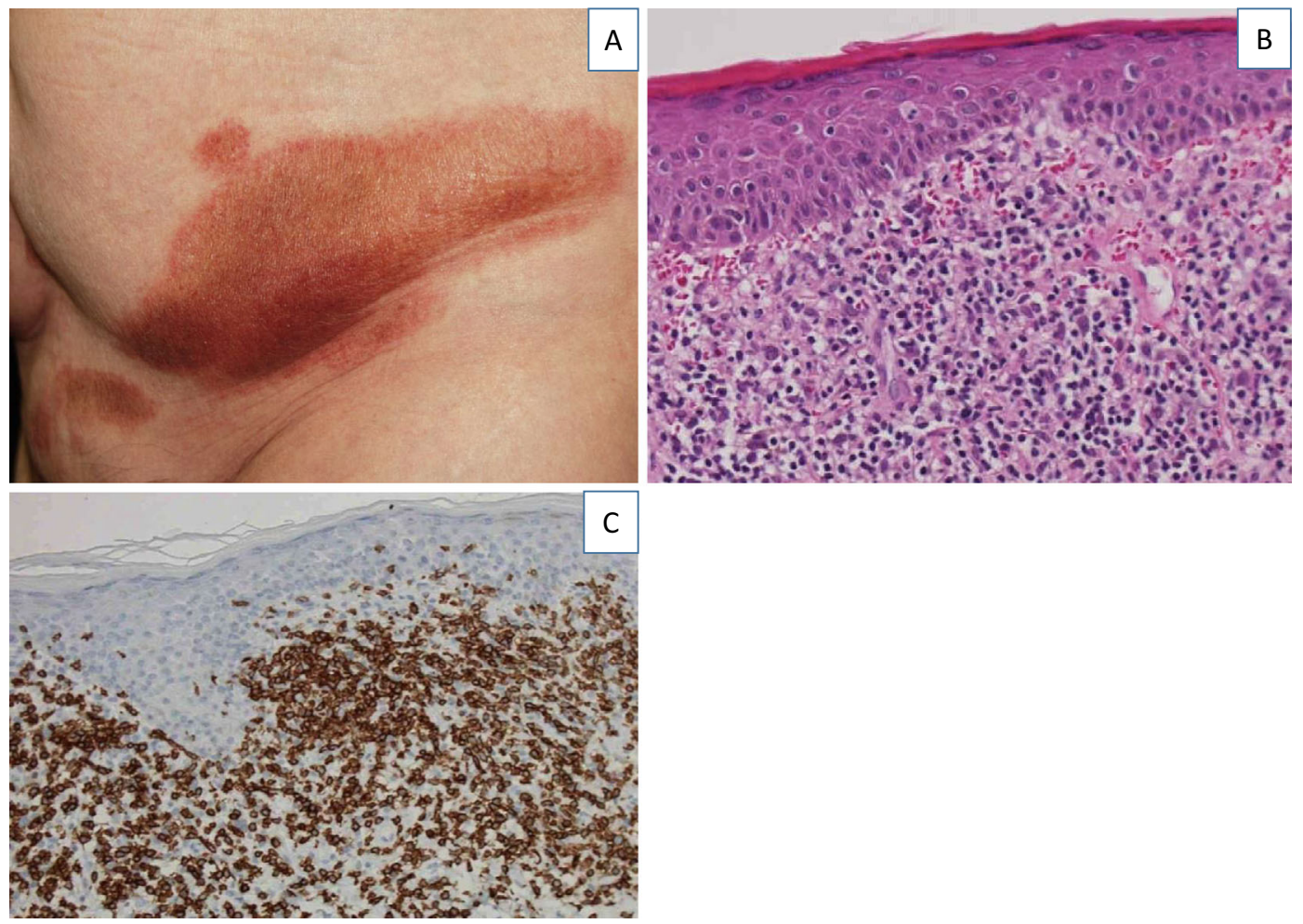

Figure 4. Case 2, mycosis fungoides. (A) Erythematous skin lesion in the left lower abdomen that extends to the inguinal region. (B) A histological analysis of specimens derived from the skin lesion showing small and medium-sized atypical lymphoid cells with visible epidermotropism (H\&E staining $\times 100)$. (C) The atypical lymphoid cells infiltrating the skin were positive for CD3 (CD3 stain, $\times 100)$.

\section{Discussion}

MF is the most common cutaneous T-cell lymphoma and is characterized by skin lesions developing over years to decades that slowly progress from localized plaques to diffuse erythroderma patches and sometimes thicker tumor lesions (10). The outcomes are largely dependent on the extent of the skin, blood, lymph node, and visceral organ involvement, with advanced age, systemic extension, and increased LDH levels being frequently associated with a poor survival (11).

MF is notorious for its association with an increased risk for the development of secondary malignancies compared with healthy population $(4,5,12)$. The coexistence of MF and B-cell malignancies in the same patient has been described before as either isolated case reports or relatively large case series $(4,12)$; the time elapsed between the onset of B-cell malignancies may range from 4 to 22 years $(12,13)$. In patients with $\mathrm{MF}$, the risk of developing secondary Hodgkin disease is greatest in males and older patients, whereas secondary non-Hodgkin lymphoma appears to favor younger patients (4).

The mechanisms underlying such an increase in the rate of developing second malignancies is unclear, although several potential mechanisms have been proposed, including an inherent potential of MF to increase the risk of malignant transformation or a consequence of the therapies utilized for MF. For example, patients with secondary malignancies preceded by MF were often exposed to chemotherapy, phototherapy, radiation, and electron beam therapy. These therapies have the potential to generate an intense immune response, resulting in a chronic inflammatory systemic response. In addition, they induce direct or indirect DNA damage, which also contributes to the increased risk of malignant transformation $(14,15)$. Furthermore, reduced levels of vitamin $\mathrm{D}$ or possessing a risk allele of the vitamin $\mathrm{D}$ receptor gene have been reported to contribute to the pathogenesis of MF and to increase the risk of secondary malignancies (16). Data from whole-genome sequencing have also revealed the increased frequency of mutations in oncogenic genes such as P53 and JAK3 in patients with MF, which can contribute to the predisposition of these patients to other cancers (17).

Intriguingly, the frequent exposure to sunlight [ultraviolet (UV) radiation] has been linked to a reduced risk of nonHodgkin lymphoma (NHL), especially DLBCL $(18,19)$; however, in patients exposed to therapeutic UV radiation, such as UV-B radiation, an increased incidence rate of leukemia has been reported $(20,21)$. Of note, Case 1 reported here received NB-UVB therapy for MF, but Case 2 did not. In contrast, secondary AML arising in CTCL patients receiving psoralen and UV therapy has been reported, suggesting that therapies for CTCL are frequently associated with 


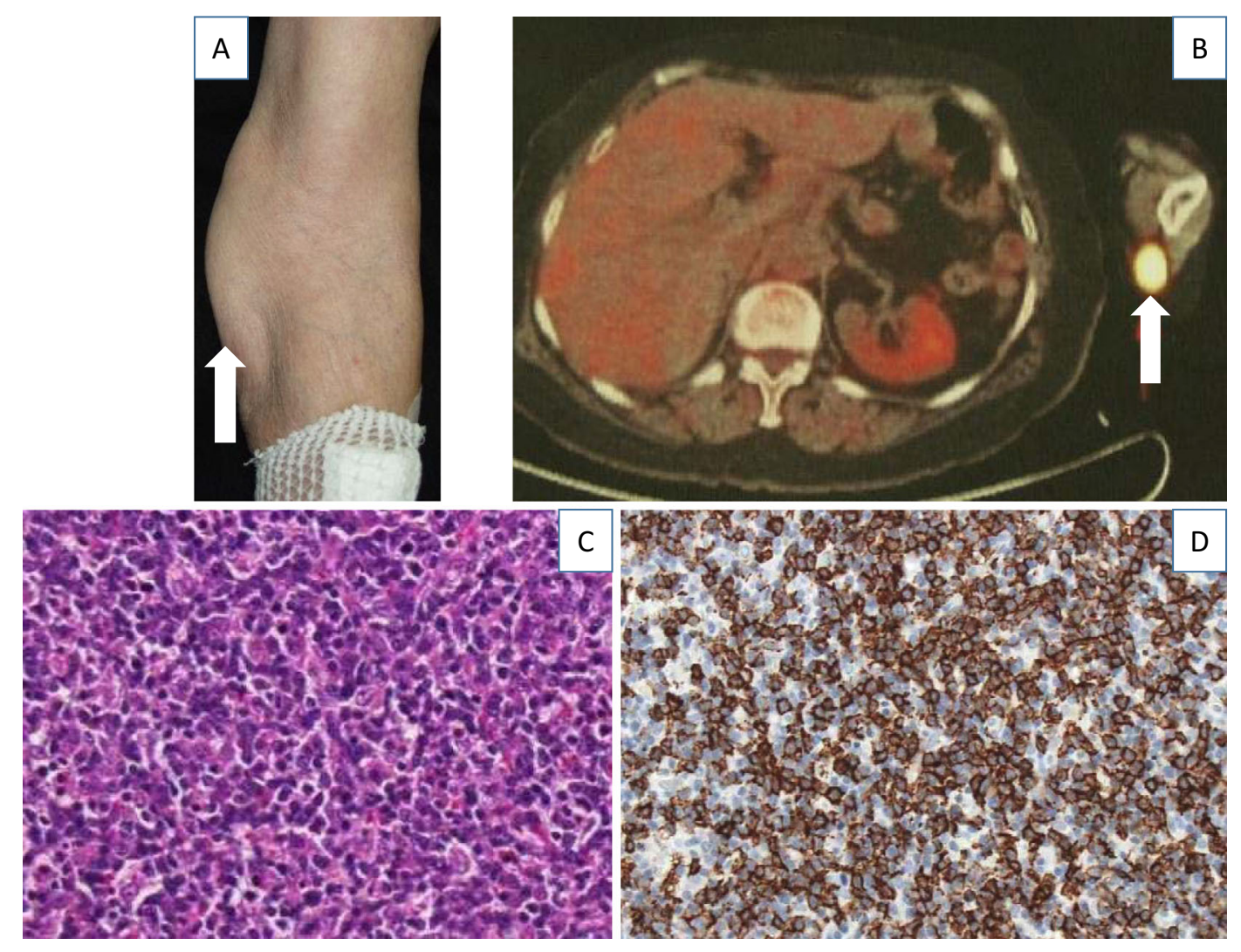

Figure 5. Case 2, DLBCL cubital lymph node. (A) A photograph of the patient's left arm showing swelling of the cubital fossa (white arrow) due to an enlarged lymph node. (B) An enlarged lymph node with the strong accumulation of FDG (SUVmax. 6.92) as observed on PET-CT (white arrow). The spleen is not visible due to splenectomy 10 years earlier. (C) Hematoxylin and Eosin staining of the lymph node-derived specimen showing abundant and diffuse proliferation of malignant lymphocytes $(\times 400)$. (D) An immunohistochemistry analysis of the lymph node specimen showing that the diffuse proliferating large cells were positive for $\mathrm{CD20}$, consistent with the diagnosis of DLBCL $(\times 400)$.

the development of AML (22).

Mixed phenotype acute leukemia is a rare and difficult-totreat hematologic malignancy with co-expression of at least two cell lineages and in rare cases involving three lineages. A distinctive characteristic of this disease entity is the presence of the undifferentiated leukemia cells, featuring at least two cell lineages at the diagnosis (23). This is in contrast to the two cases presented here, which characteristically showed the consecutive emergence over time of tumor cells derived from different cell lineages in patients who presented with MF.

A clonal analysis of the hematopoietic stem cell compartment suggests that recurrent TET2 mutations can be early events in hematologic cancers, and recent reports have shown that TET2 mutations are detectable not only in substantial myeloid malignancies but also in B-/T-cell lymphomas (24). AML/myelodysplastic syndrome (MDS) arising in patients with lymphoma were found to carry the same TET2 mutation as the preceding lymphoma, indicating a common cell of origin (25). It was recently reported that TET2 protein expression may be an independent predictor for progressive disease and death in patients with MF (26), and
TET2 mutations were reported in 12 of 100 patients with DLBCL, with 7\% carrying loss-of-function and 5\% carrying missense mutations (27). Furthermore, the presence of TET2 mutations has been documented in AML patients with normal cytogenetics (28).

The chromosomal analyses of bone marrow in the cases presented here revealed a normal karyotype without the loss of the long arms of chromosomes 5 and/or 7 at the diagnosis of AML, and the chimeric DNA quantitative determinations of MLL-ELL, MLL-AF6, and MLL-AF9 were also negative in Case 1. Notably, in this case, the chromosome analysis of spleen specimens revealed hyperdiploidy with complex anomaly (Table); regrettably, however, the chromosomal analysis of skin specimens from this patient was not available, so whether or not the MF cells carry such chromosomal aberrations is unknown.

Our in-deep mutation analysis of TET2 showed no somatic mutations in this gene in the malignant tissues that were analyzed in Case 1. Consequently, in this case and based on the mutational status of TET2 gene, there is no evidence to support the assumption that MF and DLBCL developed from a common cell of origin. Although it may 
Table. The Clinical Courses of Two Cases with Three Hematologic Malignancies and the Periods of the Diagnosis from the First Malignancy to the Next Malignancy.

\begin{tabular}{|c|c|c|}
\hline & Case 1 & Case 2 \\
\hline age & 77 & 77 \\
\hline gendar & male & female \\
\hline 1st malignancy & MF & DLBCL(spleen) \\
\hline stage & $1 \mathrm{~B}$ & $1 \mathrm{~S}$ \\
\hline chromosome & ND & ND \\
\hline treatment & NB-UVB & chemotherapy \\
\hline & topical corticosteroid & splenectomy \\
\hline periods (months)* & 0 & 0 \\
\hline 2nd malignancy & DLBCL(spleen) & MF \\
\hline stage & $1 \mathrm{~S}$ & $1 \mathrm{~A}$ \\
\hline chromosome & $* *$ & ND \\
\hline treatment & $\begin{array}{l}\text { Rituximab+ } \\
\text { chemotherapy }\end{array}$ & topical corticosteroid \\
\hline periods (months)* & 12 & 120 \\
\hline 3rd malignancy & AML(M4) & DLBCL(foosa cubitlis) \\
\hline stage & - & $1 \mathrm{~A}$ \\
\hline chromosome & $46 X Y$ & ND \\
\hline treatment & chemotherapy & $\begin{array}{l}\text { Rituximab+chemotherapy } \\
\text { splenectomy }\end{array}$ \\
\hline periods (months)* & 30 & 122 \\
\hline 4th malignancy & & AML(M4) \\
\hline stage & & - \\
\hline chromosome & & $46 X Y$ \\
\hline treatment & & chemotherapy \\
\hline periods (months)* & & 125 \\
\hline \multicolumn{3}{|c|}{$\begin{array}{l}\text { The period was up to the diagnosis of each malignancy from the diagnosis of first } \\
\text { malignancy. } \\
* * 92, \mathrm{XX},-\mathrm{Y},-\mathrm{Y},+1, \operatorname{add}(1)(\mathrm{p} 11) \times 2,+5,-6,-6, \operatorname{add}(6)(\mathrm{q} 13),+7,-9,-10,-13,-14 \text {, } \\
15,-17,+7 \operatorname{mar}<1 / 4>46 \mathrm{XY}<3 / 4>\end{array}$} \\
\hline
\end{tabular}

be necessary to rule out the presence or absence of B-cell rearrangement in the CTCL cells from this case, it is very likely that the AML nor the DLBCL in Case 1 were not therapy-related malignancies but rather two different hematological malignancies coexisting in the same patient.

The spleen is an organ with important immunological and hematological functions, including antibody production; the maturation, activation and storage of lymphocytes; iron recycling; and the mechanical filtration of blood, which is critical for removing damaged and aged blood cells and for the clearance of bacteria and other pathogens (29). Recent studies have revealed an increased risk for developing some malignancies in patients who have undergone splenectomy. In a large study of 8,149 splenectomized patients, the authors reported an increased risk of developing solid cancers (odd ratios =1.3-1.9) and hematologic malignancies in splenectomized patients, including non-Hodgkin lymphoma, Hodgkin lymphoma, multiple myeloma, AML, chronic lymphocytic leukemia, chronic myeloid leukemia, and any leukemia (odd ratios =1.8-6.0). These patients also had an increased risk of death due to any cancer, including that of the liver, pancreas, and lung; non-Hodgkin lymphoma; Hodgkin lym- phoma; and any leukemia (odd ratios $=1.3-4.7$ ). Of note, the risks were significantly elevated at more than 10 years after splenectomy (30). However, whether or not splenectomy performed as part of the treatment for DLBCL in the two cases reported here contributed to the development of secondary malignancies in these patients is unclear.

The patients described in the present report were treated with rituximab. Isolated case reports and anecdotal experience have raised the question of whether or not treatment with rituximab increases the risk of second primary malignancies (31). However, a recent meta-analysis found no predisposition to second primary malignancies among NHL survivors exposed to rituximab at a median follow-up of six years (32).

An important factor that may predispose patients with MF to develop secondary malignancies is the relative immunodeficiency that patients with cutaneous lymphoma, such as MF and Sézary syndrome (SS), typically develop during disease progression (33). In these lymphomas, malignant $\mathrm{T}$ cells appear to promote immunodeficiency in an attempt to avoid antitumor immunity. Although the underlying mechanisms remains unclear, it has become evident that a subset of 
highly immunosuppressive regulatory $\mathrm{T}$ cells (Tregs) emerge from the infiltrating malignant $\mathrm{T}$ cells pool $(15,33)$. Therefore, this immunosuppressive-like state may not only determine the increased risk of severe infections typical of patients with MF but also be a key factor that favors the growth other malignant cells. Interestingly, Treg clones appears to play a central role in the pathogenesis and progression of MF and SS, as demonstrated by the promising clinical response observed in patients treated with the anti-CC chemokine receptor 4 monoclonal antibody mogamulizumab, which depletes malignant Treg clones (34).

In summary, we herein report two rare cases of three hematological malignancies featuring two lymphoid lineages and a myeloid lineage that emerged over a relatively short period of time. To our knowledge, these are the first such cases reported in the literature. Although whether or not the three diseases coexisting in these two cases are etiologically associated remains unclear, searching for potential carcinogenic markers in specimens derived from each tumor using new screening technologies, including multiparameter cellular analyses, proteomic studies, and extensive genetic studies such as single nucleotide polymorphism (SNP) array and NGS techniques, may help clarify the pathogenesis of these diseases. The identification of such molecular events can be utilized as surrogate biomarkers of malignant transformation for the early detection of these malignancies.

The authors state that they have no Conflict of Interest (COI).

\section{References}

1. Girardi M, Heald PW, Wilson LD. The pathogenesis of mycosis fungoides. N Engl J Med 350: 1978-1988, 2004.

2. Oluwole OO, Zic JA, Douds JJ, Ann Thompson M, Greer JP. Cutaneous manifestations and management of hematologic neoplasms. Semin Oncol 43: 370-383, 2016.

3. Chang D, Zerbini MC, Sotto MN, Siqueira SA, Sanches JA. Evaluation of the 2008 world health organization classification for non-mycosis fungoides, non- Sézary syndrome T/NK-cell lymphomas with primary cutaneous involvement. J Cutan Pathol 2015 (Epub ahead of print).

4. Amber KT, Bloom R, Nouri K. Second primary malignancies in CTCL patients from 1992 to 2011: A seer-based, population-based study evaluating time from ctcl diagnosis, age, sex, stage, and CD30+ subtype. Am J Clin Dermatol 17: 71-77, 2016.

5. Brownell I, Etzel CJ, Yang DJ, Taylor SH, Duvic M. Increased malignancy risk in the cutaneous T-cell lymphoma patient population. Clin Lymphoma Myeloma 8: 100-105, 2008.

6. Jiang M, Bennani NN, Feldman AL. Lymphoma classification update: B-cell non-Hodgkin lymphomas. Expert Rev Hematol 10: 405-415, 2017

7. Lossos IS, Gascoyne RD. Transformation of follicular lymphoma. Best Pract Res Clin Haematol 24: 147-163, 2011.

8. Espinoza JL, Elbadry MI, Taniwaki M, et al. The simultaneous inhibition of the mTOR and mapk pathways with gnetin-c induces apoptosis in acute myeloid leukemia. Cancer Lett 400: 127-136, 2017.

9. Stieglitz E, Taylor-Weiner AN, Chang TY, et al. The genomic landscape of juvenile myelomonocytic leukemia. Nat Genet 47: 1326-1333, 2015
10. Cerroni L. Past, present and future of cutaneous lymphomas. Semin Diagn Pathol 34: 3-14, 2017.

11. Agar NS, Wedgeworth E, Crichton S, et al. Survival outcomes and prognostic factors in mycosis fungoides/Sézary syndrome: Validation of the revised international society for cutaneous lymphomas/ european organisation for research and treatment of cancer staging proposal. J Clin Oncol 28: 4730-4739, 2010.

12. Barzilai A, Trau H, David M, et al. Mycosis fungoides associated with B-cell malignancies. Br J Dermatol 155: 379-386, 2006.

13. Chan BC, Stefanato CM, Moonim M, et al. Diffuse large B-cell lymphoma developing in erythrodermic cutaneous T-cell lymphoma: A case series. Br J Dermatol 177: e138-e140, 2017.

14. Bagot M. New targeted treatments for cutaneous T-cell lymphomas. Indian J Dermatol 62: 142-145, 2017.

15. Wilcox RA. Cutaneous T-cell lymphoma: 2016 update on diagnosis, risk-stratification, and management. Am J Hematol 91: 151165, 2016.

16. Rasheed H, Hegazy RA, Gawdat HI, et al. Serum vitamin D and vitamin $\mathrm{D}$ receptor gene polymorphism in mycosis fungoides patients: A case control study. PLoS One 11: e0158014, 2016.

17. McGirt LY, Jia P, Baerenwald DA, et al. Whole-genome sequencing reveals oncogenic mutations in mycosis fungoides. Blood 126: 508-519, 2015.

18. Smedby KE, Hjalgrim $H$, Melbye $M$, et al. Ultraviolet radiation exposure and risk of malignant lymphomas. J Natl Cancer Inst 97: 199-209, 2005

19. Chang ET, Canchola AJ, Cockburn M, et al. Adulthood residential ultraviolet radiation, sun sensitivity, dietary vitamin $\mathrm{D}$, and risk of lymphoid malignancies in the california teachers study. Blood 118: 1591-1599, 2011.

20. Mohr SB, Garland CF, Gorham ED, Grant WB, Garland FC. Ultraviolet $\mathrm{B}$ and incidence rates of leukemia worldwide. Am J Prev Med 41: 68-74, 2011.

21. Uehara M, Takahashi K, Hoshuyama T, Pan G, Feng Y. Geographical correlation between ambient UVB level and mortality risk of leukemia in Japan. Environ Res 92: 78-84, 2003.

22. Kwong YL, Au WY, Ng MH, Chan LC, Au TS. Acute myeloid leukemia following psoralen with ultraviolet a therapy: a fluorescence in situ hybridization study. Cancer Genet Cytogenet 99: 1113, 1997.

23. Wolach O, Stone RM. Mixed-phenotype acute leukemia: current challenges in diagnosis and therapy. Curr Opin Hematol 24: 139145, 2017.

24. Lindsley RC, Mar BG, Mazzola E, et al. Acute myeloid leukemia ontogeny is defined by distinct somatic mutations. Blood 125: 1367-1376, 2015.

25. Kubuki Y, Yamaji T, Hidaka T, et al. Tet2 mutation in diffuse large b-cell lymphoma. J Clin Exp Hematop 56: 145-149, 2017.

26. Lemonnier F, Couronné L, Parrens M, et al. Recurrent TET2 mutations in peripheral T-cell lymphomas correlate with TFH-like features and adverse clinical parameters. Blood 120: 1466-1469, 2012.

27. Asmar F, Punj V, Christensen J, et al. Genome-wide profiling identifies a DNA methylation signature that associates with TET2 mutations in diffuse large B-cell lymphoma. Haematologica 98: 1912-1920, 2013.

28. Fernandez-Mercado M, Yip BH, Pellagatti A, et al. Mutation patterns of 16 genes in primary and secondary acute myeloid leukemia (AML) with normal cytogenetics. PLoS One 7: e42334, 2012.

29. Buffet PA, Safeukui I, Deplaine G, et al. The pathogenesis of plasmodium falciparum malaria in humans: insights from splenic physiology. Blood 117: 381-392, 2011.

30. Kristinsson SY, Gridley G, Hoover RN, Check D, Landgren O. Long-term risks after splenectomy among 8,149 cancer-free American veterans: a cohort study with up to 27 years follow-up. Haematologica 99: 392-398, 2014. 
31. Aksoy S, Arslan C, Harputluoglu H, Dizdar O, Altundag K. Malignancies after rituximab treatment: just coincidence or more? J BUON 16: 112-115, 2011.

32. Fleury I, Chevret S, Pfreundschuh M, et al. Rituximab and risk of second primary malignancies in patients with non-Hodgkin lymphoma: a systematic review and meta-analysis. Ann Oncol 27: 390-397, 2016.

33. Krejsgaard T, Odum N, Geisler C, et al. Regulatory $\mathrm{T}$ cells and immunodeficiency in mycosis fungoides and Sézary syndrome. Leukemia 26: 424-432, 2012.
34. Duvic M, Pinter-Brown LC, Foss FM, et al. Phase $1 / 2$ study of mogamulizumab, a defucosylated anti-CCR4 antibody, in previously treated patients with cutaneous T-cell lymphoma. Blood 125: 1883-1889, 2015.

The Internal Medicine is an Open Access article distributed under the Creative Commons Attribution-NonCommercial-NoDerivatives 4.0 International License. To view the details of this license, please visit (https://creativecommons.org/licenses/ by-nc-nd/4.0/).

(C) 2018 The Japanese Society of Internal Medicine Intern Med 57: 1445-1453, 2018 